\title{
Co-administration of methyl donors along with guanidinoacetic acid reduces the incidence of hyperhomocysteinaemia compared with guanidinoacetic acid administration alone
}

\author{
Sergej M. Ostojic ${ }^{1,2 *}$, Barbara Niess ${ }^{3}$, Marko Stojanovic ${ }^{1,2}$ and Milos Obrenovic ${ }^{1}$ \\ ${ }^{1}$ Exercise Physiology Laboratory, Center for Health, Exercise and Sport Sciences, Stari DIF, Deligradska 27, \\ Belgrade 11000, Serbia \\ ${ }^{2}$ Faculty of Sport Sciences and Tourism, Metropolitan University, Novi Sad, Serbia \\ ${ }^{3}$ AlzChem AG, Trostberg, Germany \\ (Submitted 20 September 2012 - Final revision received 6 December 2012 - Accepted 6 December 2012 - First published online 28 January 2013)
}

\section{Abstract}

Guanidinoacetic acid (GAA) is the natural biosynthetic precursor of creatine, in a metabolic reaction that requires only a methyl group transfer. The use of GAA as a food additive for restoring creatine load in human tissues is rather unexplored and data on efficacy and safety are limited. In particular, an increase in serum homocysteine after GAA administration can be regarded as critical and should be prevented. The present study evaluated the effects of orally administered GAA with and without methyl group donors on serum and urine creatine concentrations, and the occurrence of adverse events during an intervention in healthy human subjects. A total of twenty male and female volunteers were randomised in a double-blind design to receive either GAA ( $2.4 \mathrm{~g} / \mathrm{d})$ or GAA with methyl donors $\left(2.4 \mathrm{~g} / \mathrm{d}\right.$ of GAA and $1.6 \mathrm{~g} / \mathrm{d}$ of betaine $\mathrm{HCl}, 5 \mu \mathrm{g} / \mathrm{d}$ of vitamin $\mathrm{B}_{12}, 10 \mathrm{mg} / \mathrm{d}$ of vitamin $\mathrm{B}_{6}$ and $600 \mu \mathrm{g} / \mathrm{d}$ of folic acid) by oral administration for 8 weeks. Serum and urine creatine increased significantly from before to after administration in both groups $(P<0 \cdot 001)$. The proportion of participants who reported minor adverse events was $33.3 \%$ in the GAA group, and $10.0 \%$ in the GAA with methyl donors group $(P=0.30)$. Hyperhomocysteinaemia was found in $55.6 \%$ of participants supplemented with GAA, while no participant experienced hyperhomocysteinaemia in the group supplemented with GAA and methyl donors $(P=0 \cdot 01)$. In summary, both interventions strongly influenced creatine metabolism, resulting in a significant increase in fasting serum creatine. The concomitant supplementation of methyl donors along with GAA largely precluded the elevation of serum homocysteine caused by GAA administration alone.

\section{Key words: Betaine: Folic acid: Clinical enzymes: Homocysteine}

Guanidinoacetic acid (GAA) occurs naturally in the human body and is the direct biosynthetic precursor of creatine, the latter playing an important role as an energy carrier and mediator in the cell ${ }^{(1,2)}$. GAA itself along with L-ornithine is formed in an enzyme-catalysed step from L-arginine and glycine, mainly in the kidney and pancreas. The second enzyme in the pathway is guanidinoacetate $N$-methyltransferase, which catalyses the transfer of a methyl group from $S$-adenosylmethionine to GAA to form creatine and $S$-adenosylhomocysteine. Methylation takes place mainly in the liver. Creatine is then released from the liver into the circulation where it can be taken up, via a specific transporter, by various tissues ${ }^{(3)}$. Creatine synthesis from GAA is considered to be a major user of labile methyl groups from $S$-adenosylmethionine and could affect homocysteine metabolism, although studies testing the effect of creatine supplementation on plasma homocysteine concentration have shown inconsistent results ${ }^{(4-7)}$.

The metabolic and clinical effects of oral application of GAA alone or with methyl donors (e.g. betaine, choline, serine) have been evaluated in several studies. According to research in rodents ${ }^{(8)}$, short-term oral administration of GAA increases the plasma level of creatine to a similar extent as an equimolar dose of creatine, thus affecting creatine metabolism. The increase in plasma creatine was accompanied by a $49 \%$ higher plasma homocysteine level compared with control animals. In a few animal studies, GAA has been used as a methyl group acceptor to induce experimental hyperhomocysteinaemia by augmenting the conversion of $S$-adenosylmethionine to $S$-adenosylhomocysteine and then to homocysteine ${ }^{(9-11)}$. Often, hyperhomocysteinaemia can be, at least partly, corrected by promoting the homocysteine removal mechanisms

Abbreviation: GAA, guanidinoacetic acid.

*Corresponding author: Professor S. M. Ostojic, fax +381 112643 242, email sergej.ostojic@chess.edu.rs 
either through trans-sulfuration to cysteine, remethylation to methionine, or in combination ${ }^{(12,13)}$. In this context, nutrients such as betaine, choline and vitamin $\mathrm{B}_{6}$, vitamin $\mathrm{B}_{12}$, folic acid and vitamin $B_{2}$ have been extensively studied for the prevention or treatment of hyperhomocysteinaemia ${ }^{(14-16)}$. In rodents, the addition of methyl donors such as choline or betaine effectively suppressed hyperhomocysteinaemia induced by $\operatorname{GAA}^{(10,11)}$. It is usually assumed that dietary choline (after oxidation to betaine) or betaine stimulates homocysteine remethylation via betaine-homocysteine $S$-methyltransferase, but other mechanisms have also been considered ${ }^{(10)}$. Early clinical studies have revealed an improved sense of wellbeing, less fatigue and enhanced work capacity after oral administration of GAA and the methyl donor betaine in patients with heart disease and disability resulting from acute anterior poliomyelitis ${ }^{(17-19)}$. In these studies, betaine was supplied in a three- to fivefold molar excess in relation to GAA. The transformation of GAA to creatine was monitored via the excretion of creatine in the urine. The combination was effective as adjunctive therapy in the management of anxiety-fatigue syndrome ${ }^{(20)}$, but did not show an advantage in the treatment of arthritis and motor neuron disease ${ }^{(21,22)}$. Independently of the therapeutic success, the studies found no side effects except for occasional gastrointestinal disturbances, although information about the effect of GAA and betaine on clinical markers of health status is limited. Higgins et al. ${ }^{(21)}$ observed no change in serum protein, urinary excretion of $\mathrm{Na}, \mathrm{K}, \mathrm{Ca}$ and $\mathrm{P}$, and serum alkaline phosphatase after the administration of GAA and betaine. Recently, we have shown that the daily administration of $2.4 \mathrm{~g}$ GAA alone for 6 weeks to young healthy volunteers had a significant impact on creatine metabolism by increasing serum and urine creatine and creatinine, but was accompanied by a $28 \%$ increase in fasting plasma homocysteine (SM Ostojic, B Niess and MD Stojanovic, unpublished results). Other disturbances of clinical markers were not observed and the occurrence of self-reported adverse events was rather low. Nevertheless, total homocysteine is a possible risk factor for CVD and an increase may be regarded as critical. The aims of the present study were: (1) to investigate whether oral administration of GAA for 8 weeks with and without methyl donors affected creatine metabolism differently in young healthy volunteers; (2) to evaluate the effects of both interventions on fasting total plasma homocysteine; (3) to monitor the occurrence of clinical and biochemical adverse events during the intervention.

\section{Methods}

\section{Subjects}

A total of twenty healthy volunteers (age 22.6 (SD 3.0) years, weight $70.6(\mathrm{SD} \mathrm{12.3)} \mathrm{kg}$ and height $174.2(\mathrm{SD} 7.7) \mathrm{cm}$; ten men and ten women) were recruited to participate in the present study. The study was conducted according to the guidelines laid down in the Declaration of Helsinki, and all procedures involving human subjects were approved by the Center Ethical Committee (approval no. 176/11-EUTC-49). Written informed consent was obtained from all subjects.
Inclusion criteria were as follows: age between 18 and 30 years; no pre-existing clinical or medical conditions; moderately physically active participants; no use of any dietary supplements within the $60 \mathrm{~d}$ before the study. The present trial has been registered at ClinicalTrials.gov (identification no. NCT01371357).

\section{Study protocol}

A double-blind, GAA-controlled, randomised trial was performed to assess the effect of the oral administration of GAA alone when compared with GAA with methyl donors on serum and urine creatine, creatinine and GAA, total plasma homocysteine and the rate of adverse events. Subjects were assigned into two groups receiving either $2.4 \mathrm{~g} \mathrm{GAA} / \mathrm{d}$, or $2.4 \mathrm{~g} / \mathrm{d}$ of GAA with $1.6 \mathrm{~g} / \mathrm{d}$ of betaine $\mathrm{HCl}, 5 \mu \mathrm{g} / \mathrm{d}$ of vitamin $\mathrm{B}_{12}, 10 \mathrm{mg} / \mathrm{d}$ of vitamin $\mathrm{B}_{6}$ and $600 \mu \mathrm{g} / \mathrm{d}$ of folic acid. The treatment formulation was provided by AlzChem AG in prepacked powder form. Appearance, taste and powder mass were standardised by the addition of citric acid, maltodextrin, lemon flavour and sucralose to be undistinguishable from each other. The daily dose was taken in two portions $(1.2 \mathrm{~g}$ GAA at each dose) and the participants were instructed to dissolve each dose in a drink right before consumption. Of these doses, one was taken in the morning upon waking before breakfast and the other in the evening before the last meal. Both formulations were administered for 8 weeks to the participants. The twenty volunteers were randomly assigned to receive GAA (ten participants) or GAA with methyl donors (ten participants), and women had an equal probability of assignment to the groups. All subjects who started the treatment were included in the intention-to-treat population.

\section{Blood and urine analyses}

At baseline and after 8 weeks, participants provided $24 \mathrm{~h}$ urine and fasting blood samples, with analyses completed after the completion of the study. Urine was collected in a plastic bottle and the volume was measured. An aliquot $(10 \mathrm{ml})$ of the homogenised sample was stored at $-20^{\circ} \mathrm{C}$ and analysed for GAA, creatine and creatinine. Blood was collected from a radial vein in a gel vacutainer for biochemical variables, and $7 \cdot 5 \% \mathrm{~K}_{3}$-EDTA vacutainer for homocysteine. Gel vacutainers were centrifuged within the next $30 \mathrm{~min}$ at $3000 \mathrm{~g}$ for $15 \mathrm{~min}$, with serum stored at $-20^{\circ} \mathrm{C}$. The $\mathrm{K}_{3}$-EDTA tubes were centrifuged within the next $10 \mathrm{~min}$ at $3000 \mathrm{~g}$ for $10 \mathrm{~min}$. Plasma was separated, stored at $-20^{\circ} \mathrm{C}$ and analysed for homocysteine. Total plasma homocysteine was measured with a fluorescence polarisation immunoassay method (DPC Immulite 2000; Siemens AG). Hyperhomocysteinaemia was defined as total plasma homocysteine $>10 \cdot 4 \mu \mathrm{mol} / \mathrm{l}$ for women and $>11.4 \mu \mathrm{mol} / 1$ for men ${ }^{(23)}$. Creatine, GAA and creatinine in both serum and urine samples were analysed by pre-column derivatisation ${ }^{(24)}$ with a modular system (HP Agilent 1100 HPLC; Hewlett-Packard Company). Serum creatinine $>90 \mu \mathrm{mol} / 1$ in women and $>110 \mu \mathrm{mol} / 1$ in men was considered as indicative of renal damage ${ }^{(25)}$. Concerns have been raised that GAA administration may induce liver 
and/or muscle damage ${ }^{(8)}$. These changes in hepatocytes and myocytes can lead to the leakage of parenchymal (transaminases) and membranous (alkaline phosphatase) enzymes of the liver and muscle (creatine kinase) into the circulatory system. Therefore, serum activities of aspartate aminotransferase, alanine aminotransferase, alkaline phosphatase, $\gamma$-glutamyl transpeptidase and creatine kinase were analysed (RX Daytona; Randox Laboratories Limited), with abnormal findings for enzyme levels being identified according to the clinical guidelines ${ }^{(26)}$. Participants were instructed to report any adverse event of the supplementation protocol through an open-ended questionnaire for self-assessment of well-being during the study. The primary endpoint was the change in total plasma homocysteine after 8 weeks of administration. The primary safety outcome was the frequency of adverse events during the treatment period.

\section{Statistical analysis}

The required sample of ten subjects in each of the two groups was estimated with the goal of rejecting the null hypothesis if the means of the total plasma homocysteine, with equal standard deviations of $2.0 \mu \mathrm{mol} / 1$, differed by at least $1.0 \mu \mathrm{mol} / 1$ according to the Mann-Whitney $U$ test, with a type I error of 0.05 (two-sided) and $90 \%$ power. All results are expressed as means and standard deviations for continuous variables, and rates for categorical variables. The frequency of adverse event occurrence was compared between the groups using the Fisher exact probability test. Baseline characteristics of study participants were compared with the two-sample $t$ test. Two-way ANOVA with repeated measures was used to establish whether any significant differences existed between subjects' responses over time of the intervention ( $0 v .8$ weeks). Where significant differences were found, the Tukey test was employed to identify the differences. $P$ values of less than 0.05 were considered to be statistically significant.

\section{Results}

Most baseline characteristics of the study participants were similar between the two groups (Table 1). Significant differences were observed for alkaline phosphatase $(P=0 \cdot 006), \gamma$-glutamyl transpeptidase $(P=0.033)$ and creatine kinase $(P=0.046)$, but not for aspartate aminotransferase and alanine aminotransferase. However, all values for liver and muscle function were within the normal clinical range. No biochemical abnormalities at baseline were detected and values were comparable with other data obtained from this age group and population ${ }^{(27)}$.

A total of seventeen participants completed the follow-up measures after 8 weeks. No single participant was excluded during the study due to adverse events. Only one female participant from the GAA group left the study before starting the treatment due to a significant change in health status not related to the present study. During the intervention period, one female and one male participant from the GAA group were lost due to reasons not connected to the study per se. The proportion of participants who reported minor adverse
Table 1. Baseline characteristics of the study participants (Mean values and standard deviations)

\begin{tabular}{|c|c|c|c|c|c|}
\hline & \multicolumn{2}{|c|}{ GAA $(n 9)$} & \multicolumn{2}{|c|}{$\begin{array}{c}\text { GAA with } \\
\text { methyl donors } \\
(n 10)\end{array}$} & \multirow[b]{2}{*}{$P^{\star}$} \\
\hline & Mean & SD & Mean & SD & \\
\hline Age (years) & $23 \cdot 7$ & $3 \cdot 0$ & $21 \cdot 6$ & $2 \cdot 8$ & 0.134 \\
\hline Weight (kg) & $72 \cdot 6$ & $14 \cdot 3$ & 68.9 & $10 \cdot 6$ & 0.535 \\
\hline Height $(\mathrm{cm})$ & $175 \cdot 0$ & $9 \cdot 1$ & 173.4 & $6 \cdot 7$ & 0.671 \\
\hline AST (IU/I) & 23.8 & $10 \cdot 5$ & 28.0 & $9 \cdot 8$ & 0.381 \\
\hline ALT (IU/I) & $27 \cdot 0$ & 14.9 & $28 \cdot 2$ & $22 \cdot 7$ & 0.892 \\
\hline ALP (mmol/l) & $75 \cdot 6$ & $19 \cdot 1$ & $50 \cdot 9$ & $12 \cdot 9$ & 0.006 \\
\hline$\gamma-G^{\top}(I U / I)$ & $27 \cdot 7$ & 4.9 & $19 \cdot 8$ & $9 \cdot 2$ & 0.033 \\
\hline CK (IU/I) & 124.9 & $51 \cdot 2$ & $217 \cdot 1$ & $120 \cdot 2$ & 0.046 \\
\hline $\mathrm{T}$-Hcy $(\mu \mathrm{mol} / \mathrm{l})$ & $7 \cdot 2$ & $1 . \overline{8}$ & $7 \cdot 4$ & 1.5 & 0.797 \\
\hline \multicolumn{6}{|l|}{ Serum } \\
\hline Creatine $(\mu \mathrm{mol} / \mathrm{l})$ & $23 \cdot 6$ & $8 \cdot 1$ & $22 \cdot 9$ & $10 \cdot 9$ & 0.874 \\
\hline Creatinine $(\mu \mathrm{mol} / \mathrm{l})$ & $97 \cdot 0$ & 14.2 & $94 \cdot 1$ & 9.9 & 0.617 \\
\hline GAA $(\mu \mathrm{mol} / \mathrm{l})$ & 1.8 & 0.3 & 1.5 & 0.4 & 0.081 \\
\hline \multicolumn{6}{|l|}{ Urinary } \\
\hline Creatine $(\mathrm{mg} / 24 \mathrm{~h})$ & $19 \cdot 6$ & $6 \cdot 2$ & $18 \cdot 6$ & $6 \cdot 8$ & 0.742 \\
\hline Creatinine $(\mathrm{mg} / \mathrm{l})$ & $8 \cdot 3$ & 6.4 & $8 \cdot 1$ & $6 \cdot 3$ & 0.947 \\
\hline $\mathrm{GAA}(\mu \mathrm{mol} / \mathrm{l})$ & 94.1 & $47 \cdot 6$ & $122 \cdot 7$ & 32.4 & 0.152 \\
\hline
\end{tabular}

GAA, guanidinoacetic acid; AST, aspartate aminotransferase; ALT, alanine aminotransferase; ALP, alkaline phosphatase; $\gamma$-GT, $\gamma$-glutamyl transpeptidase; CK creatine kinase; T-Hcy, total plasma homocysteine.

${ }^{*} P$ values from the two-sample $t$ test.

events was $33.3 \%$ (three of nine) in the GAA intervention group, and $10.0 \%$ (one of ten) in the GAA with methyl donors group (cumulative incidence difference 23.3\%; $95 \%$ CI $55 \cdot 6,13 \cdot 8 \%, P=0 \cdot 30)$. Mild nausea was reported in two participants from the GAA group (two of nine), with a change in saliva taste, a change in body odour and stomach burning being reported once per event (one of nine). Similarly, one participant from the group supplemented with GAA and methyl donors reported an episode of frequent urination (one of ten). The frequency of reported adverse events was similar in the two groups. The rate of biochemical adverse events was low (Table 2). Hyperhomocysteinaemia was found in $55.6 \%$ of participants supplemented with GAA, whereas not a single participant from the group supplemented with GAA plus methyl donors experienced hyperhomocysteinaemia (cumulative incidence difference 55.6\%; 95\% CI 81·1, $15.5 \%, P=0 \cdot 01$ ). Proteinuria was not found in either group.

Changes in serum and urine profiles of guanidino compounds during the study are presented in Fig. 1 . The results indicated no significant treatment $x$ time interaction for serum and urinary creatine and creatinine, while a significant interaction effect was found for both serum and urine concentrations of GAA $(P<0 \cdot 05)$.

Serum and urine creatine increased significantly from before to after administration in both intervention groups $(P=0.002)$. After 8 weeks of administration, the GAA group had a similar level of serum creatine to the GAA with methyl donors group (36.9 (SD 8.9) v. 36.1 (SD 9.0) $\mu \mathrm{mol} / 1, \quad P=0.73$ ). Urinary excretion of creatine was similar in both intervention groups at post-administration $(102.0 \quad(\mathrm{SD} 181.01) \mathrm{mg} / 24 \mathrm{~h}$ for the GAA group $v$. 117.8 (SD 78.9) $\mathrm{mg} / 24 \mathrm{~h}$ for the GAA with methyl donors group, $P=0 \cdot 71$ ). Serum and urine GAA were 
Table 2. Biochemical adverse events during the study

(Number of subjects and percentages)

\begin{tabular}{|c|c|c|c|c|}
\hline \multirow[b]{2}{*}{ Events } & \multicolumn{2}{|c|}{ GAA $(n 9)$} & \multicolumn{2}{|c|}{$\begin{array}{c}\text { GAA with } \\
\text { methyl donors } \\
(n 10)\end{array}$} \\
\hline & $n$ & $\%$ & $n$ & $\%$ \\
\hline AST $>40 \mathrm{IU} / \mathrm{l} \dagger$ & 1 & $11 \cdot 1$ & 1 & $10 \cdot 0$ \\
\hline ALT > 56 IU/I $\dagger$ & 1 & $11 \cdot 1$ & 1 & $10 \cdot 0$ \\
\hline ALP $>140 \mathrm{mmol} / \mathrm{l} \dagger$ & - & - & & \\
\hline$\gamma-\mathrm{GT}>51 \mathrm{IU} / \mathrm{l} \dagger$ & - & - & & \\
\hline $\mathrm{CK}>400 \mathrm{IU} / \mathrm{I} \dagger$ & 1 & 11.1 & 3 & $30 \cdot 0$ \\
\hline Hyperhomocysteinaemiał & $5^{\star}$ & 55.6 & - & \\
\hline Elevated serum creatinine§ & 3 & $33 \cdot 3$ & 1 & $10 \cdot 0$ \\
\hline Proteinurial| & - & - & & \\
\hline
\end{tabular}

GAA, guanidinoacetic acid; AST, aspartate aminotransferase; ALT, alanine aminotransferase; ALP, alkaline phosphatase; $\gamma$-GT, $\gamma$-glutamyl transpeptidase; CK, creatine kinase.

* Values were significant in the rate of adverse events between the groups $(P<0.05)$.

†Cut-off points for enzyme levels were identified according to the clinical guidelines ${ }^{(26)}$.

$\ddagger$ Total plasma homocysteine $>10.4 \mu \mathrm{mol} / \mathrm{l}$ for women and $>11.4 \mu \mathrm{mol} / /$ for $\mathrm{men}^{(23)}$ $\S$ Serum creatinine $>90 \mu \mathrm{mol} / \mathrm{l}$ for women and $>110 \mu \mathrm{mol} / /$ for $\mathrm{men}^{(25)}$.

\|Proteinuria was defined as $2+$ or greater protein on urinalysis during the follow-up period in participants with a negative finding or trace protein at baseline.

elevated by $3 \cdot 3(95 \% \mathrm{CI} 4 \cdot 4,2 \cdot 1) \mu \mathrm{mol} / 1$, and by $19 \cdot 2(95 \% \mathrm{CI}$ $27 \cdot 4,11 \cdot 0) \mathrm{mg} / 24 \mathrm{~h}$, respectively, after 8 weeks of administration in the GAA group. Intake of GAA with methyl donors significantly increased serum GAA from 1.5 (SD 0.4 ) to 1.9 (SD $0 \cdot 3) \mu \mathrm{mol} / 1$ at post-administration $(P=0 \cdot 01)$, while urinary excretion of GAA was not affected by the intervention (17.9 (SD 6.9) $\mathrm{mg} / 24 \mathrm{~h}$ at baseline $v .28 .4(\mathrm{SD} 16.62) \mathrm{mg} / 24 \mathrm{~h}$ at post-administration, $P=0.09)$. Serum and urine creatinine were not affected by either intervention $(P>0 \cdot 05)$. Furthermore, total plasma homocysteine increased significantly from before to after administration in GAA-administered participants (7.2 (SD 1.8) v. 12.8 (SD 2.5) $\mu \mathrm{mol} / 1, P<0.001)$. There was no significant difference in total plasma homocysteine from before to after administration in the GAA plus methyl donors group $(7 \cdot 4(\mathrm{SD} 1.5)$ at baseline $v .6 \cdot 8(\mathrm{SD} 0.9) \mu \mathrm{mol} / \mathrm{l}$ at post-administration, $P=0 \cdot 30$ ).

\section{Discussion}

The present study shows a significant increase in serum creatine and urine creatine excretion in human subjects supplemented with $2.4 \mathrm{~g} / \mathrm{d}$ of GAA for 8 weeks. Creatine concentration in serum and the amount of creatine excreted in the urine were not different between the groups regardless of whether GAA was administered alone or along with methyl donors. This implies that GAA is absorbed from the gastrointestinal tract, enters the circulation and is methylated to yield creatine in healthy human subjects, independent of the presence of methyl donors. These results support those of Stead et $a{ }^{(8)}$, and Borsook \& Borsook ${ }^{(17)}$, and indicate that exogenous GAA is a source of creatine. It seems that the dietary provision of GAA drives creatine synthesis through the guanidinoacetate $N$-methyltransferase reaction. After 8 weeks of administration, GAA clearly induced elevated serum
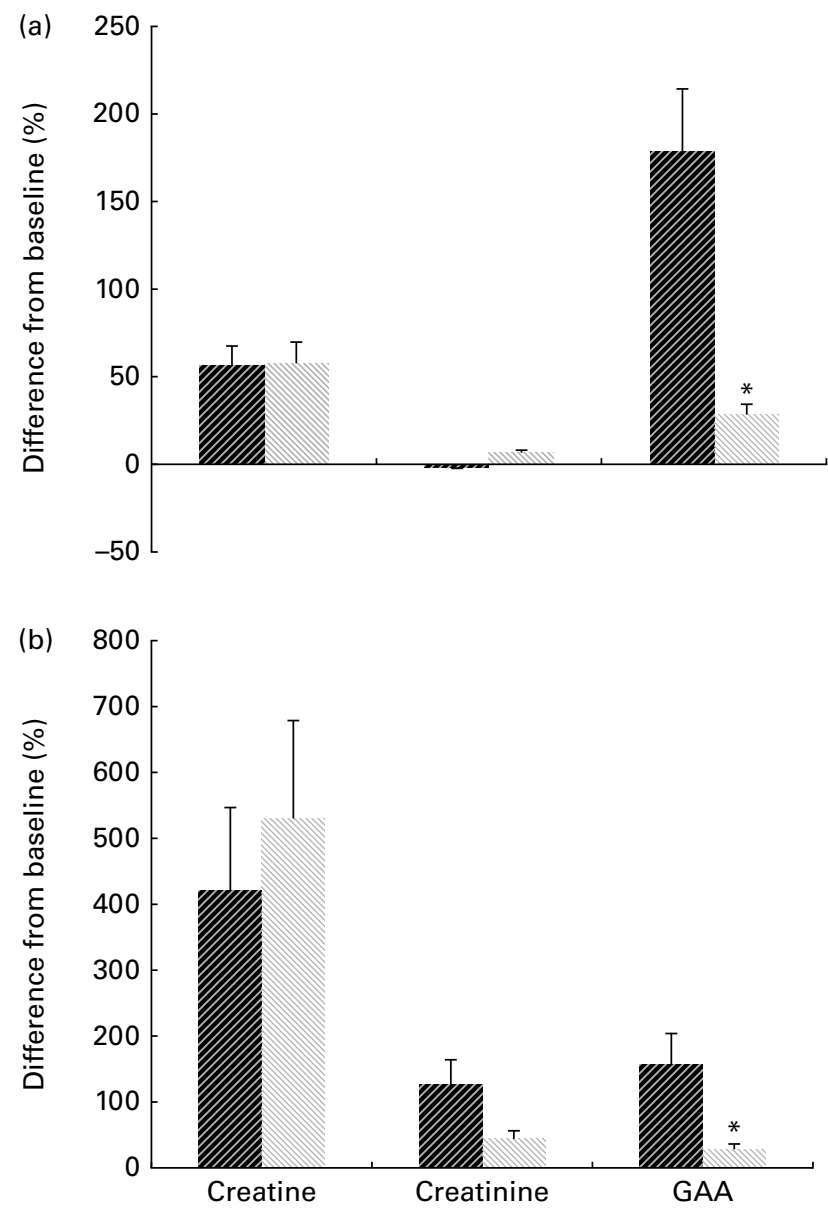

Fig. 1. Mean percentage change in (a) serum and (b) urine guanidino compounds 0 v. 8 weeks for the guanidinoacetic acid (GAA, $\mathbb{N}(n 9)$ and GAA with methyl donors groups ( $N, n$ 10). Values are means, with standard deviations represented by error bars. ${ }^{*}$ There was a significant effect for the treatment $\times$ time interaction $(P<0.05)$.

creatine by up to $50 \%$ and the fractional excretion of creatine increased up to approximately $20 \%$ of the total ingested amount of GAA (calculated as creatine). So far, it seems as if methyl donors do not affect creatine clearance dynamics (urinary clearance). During the present study, no muscle biopsies were taken; thus, the question remains to be answered whether the presence of methyl donors has an influence on the muscular accumulation of creatine.

The gastrointestinal uptake of GAA resulted in both elevated serum GAA and slightly increased urinary excretion of GAA, leading to the conclusion that excess GAA that is not methylated to creatine is excreted in the urine. Yet, serum and urinary GAA responses are rather different between sole GAA and GAA with methyl donors application. The application of GAA with methyl donors influenced the conversion of GAA by the liver (or other tissues) and/or resorption in the gut or kidney, with an attenuated rise in serum GAA after administration (perhaps due to a more efficient transformation to creatine) and a lesser rate of urinary GAA excretion, when compared with sole GAA. Additionally, low GAA excretion through the kidney for both groups during 
the present study may indicate either high utilisation and/or excretion through other organs (e.g. bile, faeces). To fully elucidate the distribution, metabolism and excretion of GAA, additional studies would be required. Although an upward trend for creatinine dynamics was noted in the present study, no differences between the groups were found for serum and urine creatinine during the intervention and the observed values for creatinine were well within the reference limits; the possibility that GAA alone or in combination with methyl donors may increase renal stress is highly unlikely.

For the present study, subjectively reported adverse events during 8 weeks of administration of different GAA formulations were rather transient, minor and clinically irrelevant, with no major disturbances of indicators of health status. The incidence of subjective adverse events and most changes in clinical markers was not different between the two groups. Yet, hyperhomocysteinaemia was found in $55.6 \%$ of participants supplemented with GAA alone, while no participant experienced hyperhomocysteinaemia in the group supplemented with GAA in combination with methyl donors. Hyperhomocysteinaemia in humans is discussed as a risk factor for CVD and neurodegenerative disease ${ }^{(28-31)}$, pregnancy complications ${ }^{(32)}$ and osteoporosis ${ }^{(33)}$. The observation of elevated serum homocysteine in participants receiving sole GAA reflects the metabolic consequence of exogenous GAA supply: exogenous GAA enters the biochemical pathway of creatine synthesis and is methylated by $S$-adenosylmethionine forming creatine and SAH. S-Adenosylhomocysteine formed during this process is subsequently hydrolysed, thus generating homocysteine, which may then enter either into a new cycle of methyl group transfer, into the trans-sulfuration pathway, or may be released into the circulation ${ }^{(12,13)}$. Previous animal studies reported that feeding a methyl group acceptor such as GAA directly drives homocysteine production ${ }^{(8)}$, which can be corrected by promoting the removal mechanisms ${ }^{(10,11)}$. We found that methyl donors and relevant cofactors such as the mixture of betaine, vitamin $\mathrm{B}_{12}$, vitamin $\mathrm{B}_{6}$ and folic acid were powerful nutrients to suppress hyperhomocysteinaemia during GAA supplementation in human subjects. Remarkably, even with a submolar amount of methyl donors (betaine in particular) compared with GAA and vitamin supply in the range of three to five times the recommended daily allowance ${ }^{(34)}$, an increase in the homocysteine level could be efficiently prevented. The average level of serum homocysteine in participants receiving GAA with methyl donors stayed below $7 \mu \mathrm{mol} / 1$ at post-administration.

The data of the present study suggest that supplementing GAA improves creatine load in healthy human subjects, with a reduced incidence of hyperhomocysteinaemia when GAA is co-administered with methyl group donors. Since creatine is heavily involved in energy metabolism, supplementation with GAA with methyl donors might be considered as a promising and safe option for general strengthening of cellular energetics in population with critical energy requirements (e.g. athletes, elderly, patients with neuromuscular diseases). Yet, implications for human health and performance are yet to be established. To fully explore applications for the nutritional or therapeutic use of oral GAA in combination with methyl donors, additional studies would be required to establish pharmacokinetic information and elucidate physiological effects of exogenous GAA via pharmacodynamics modelling.

In conclusion, it was shown that the administration of $2 \cdot 4 \mathrm{~g} / \mathrm{d}$ of GAA alone or in combination with methyl donors strongly influences creatine metabolism, with a significant increase in fasting serum creatine (up to $50 \%$ ). The concomitant administration of methyl donors together with GAA largely prevented hyperhomocysteinaemia induced by GAA alone. GAA had an acceptable safety profile with a low incidence of adverse events.

\section{Acknowledgements}

The present study was supported by grant no. BN-215E-S10 from AlzChem AG and by funding from the Serbian Ministry of Science (grant no. 175037). The authors thank the research participants for their dedication. We thank Dr Radmila Vrzic (Analiza Laboratories, Belgrade) and Dr Nadezda Majkic (Faculty of Medicine, University of Belgrade) for their assistance with the biochemical data retrieval; Mirjana Stojanovic for the statistical support; and the staff of the CHESS Exercise Physiology Laboratory, Dragoljub Veljovic, Sasa Semeredi and Milan Dimitric. S. M. O. contributed to the study concept and design. S. M. O., M. S. and M. O. were involved in the acquisition of the data. S. M. O. and M. S. conducted the analysis of the data. S. M. O. and B. N. participated in the interpretation of the data. S. M. O. and B. N. helped with the drafting of the manuscript. M. O. and B. N. were responsible for the critical revision of the manuscript for important intellectual content. S. M. O. and M. S. performed the statistical analysis. All the authors reviewed the paper, and read and approved the final manuscript. The authors declare that there are no conflicts of interest.

\section{References}

1. Walker JB (1979) Creatine: biosynthesis, regulation, function. Adv Enzymol Relat Areas Mol Biol 50, 177-242.

2. Wyss M \& Kaddurah-Daouk R (2000) Creatine and creatinine metabolism. Physiol Rev 80, 1107-1213.

3. Snow RJ \& Murphy RM (2001) Creatine and the creatine transporter: a review. Mol Cell Biochem 224, 169-181.

4. Steenge GR, Verhoef P \& Greenhaff PL (2001) The effect of creatine and resistance training on plasma homocysteine concentration in healthy volunteers. Arch Intern Med $\mathbf{1 6 1}$ 1455-1456.

5. Korzun WJ (2004) Oral creatine supplements lower plasma homocysteine concentrations in humans. Clin Lab Sci 17, 102-106

6. Taes YE, Delanghe JR, De Bacquer D, et al. (2004) Creatine supplementation does not decrease total plasma homocysteine in chronic hemodialysis patients. Kidney Int $\mathbf{6 6}$ 2422-2428.

7. Deminice R, Portari GV, Vannucchi H, et al. (2009) Effects of creatine supplementation on homocysteine levels and lipid peroxidation in rats. BrJ Nutr 102, 110-116.

8. Stead LM, Au KP, Jacobs RL, et al. (2001) Methylation demand and homocysteine metabolism: effects of dietary 
provision of creatine and guanidinoacetate. Am J Physiol Endocrinol Metab 281, E1095-E1100.

9. Fukada S, Setoue M, Morita T, et al. (2006) Dietary eritadenine suppresses guanidinoacetic acid-induced hyperhomocysteinemia in rats. J Nutr 136, 2797-2802.

10. Setoue M, Ohuchi S, Morita T, et al. (2008) Hyperhomocysteinemia induced by guanidinoacetic acid is effectively suppressed by choline and betaine in rats. Biosci Biotechnol Biochem 72, 1696-1703.

11. Ohuchi S, Matsumoto Y, Morita T, et al. (2008) High-casein diet suppresses guanidinoacetic acid-induced hyperhomocysteinemia and potentiates the hypohomocysteinemic effect of serine in rats. Biosci Biotechnol Biochem 72, 3258-3264.

12. Selhub J (1999) Homocysteine metabolism. Annu Rev Nutr 19, 217-246.

13. Finkelstein JD (1998) The metabolism of homocysteine: pathways and regulation. Eur J Pediatr 157, S40-S44.

14. Craig SAS (2004) Betaine in human nutrition. Am J Clin Nutr 80, 539-549.

15. Ueland PM (2011) Choline and betaine in health and disease. $J$ Inherit Metab Dis 34, 3-15.

16. Clarke R, Halsey J, Lewington S, et al. (2010) Effects of lowering homocysteine levels with B vitamins on cardiovascular disease, cancer, and cause-specific mortality. Arch Intern Med 170, 1622-1631.

17. Borsook ME \& Borsook H (1951) Treatment of cardiac decompensation with betaine and glycocyamine. Ann West Med Surg 5, 830-855.

18. Graybiel A \& Patterson CA (1951) Use of betaine and glycocyamine in the treatment of patients with heart disease: preliminary report. Ann West Med Surg 5, 863-875.

19. Borsook ME, Billig HK \& Golseth JG (1952) Betaine and glycocyamine in the treatment of disability resulting from acute anterior poliomyelitis. West Med Surg 6, 423-427.

20. Dixon HH, Dickel HA, Shanklin JG, et al. (1954) Therapy in anxiety states and anxiety complicated by depression. West $J$ Surg Obstet Gynecol 62, 338-341.

21. Higgins AR, Harper HA, Kline EF, et al. (1952) Effects of creatine precursors in arthritis: clinical and metabolic study of glycocyamine and betaine. Calif Med 77, 14-18.

22. Liversedge LA (1956) Glycocyamine and betaine in motor-neurone disease. Lancet 271, 1136-1138.
23. Selhub J, Jacques PF, Rosenberg IH, et al. (1999) Serum total homocysteine concentrations in the third National Health and Nutrition Examination Survey (1991-1994): population reference ranges and contribution of vitamin status to high serum concentrations. Ann Intern Med 131, 331-339.

24. Carducci C, Birarelli M, Leuzzi V, et al. (2002) Guanidinoacetate and creatine plus creatinine assessment in physiologic fluids: an effective diagnostic tool for the biochemical diagnosis of arginine:glycine amidinotransferase and guanidinoacetate methyltransferase deficiencies. Clin Chem $\mathbf{4 8}$, $1772-1778$.

25. Ceriotti F, Boyd JC, Klein G, et al. (2008) Reference intervals for serum creatinine concentrations: assessment of available data for global application. Clin Chem 54, 559-566.

26. Castagna J (editor) (2003) Handbook of Diagnostic Tests, 3rd ed. Philadelphia, PA: Lippincott Williams \& Wilkins.

27. Pettersson J, Hindorf U, Persson P, et al. (2008) Muscular exercise can cause highly pathological liver function tests in healthy men. Br J Clin Pharmacol 65, 253-259.

28. Stanger O, Herrmann W, Pietrzik K, et al. (2004) Clinical use and rational management of homocysteine, folic acid, and $\mathrm{B}$ vitamins in cardiovascular and thrombotic diseases. Z Kardiol 93, 439-453.

29. Stanger O, Fowler B, Piertzik K, et al. (2009) Homocysteine, folate and vitamin $B_{12}$ in neuropsychiatric diseases: review and treatment recommendations. Expert Rev Neurother 9, $1393-1412$.

30. Ciaccio M \& Bellia C (2010) Hyperhomocysteinemia and cardiovascular risk: effect of vitamin supplementation in risk reduction. Curr Clin Pharmacol 5, 30-36.

31. Smulders YM \& Blom HJ (2011) The homocysteine controversy. J Inherit Metab Dis 34, 93-99.

32. Nelen WL (2001) Hyperhomocysteinaemia and human reproduction. Clin Chem Lab Med 39, 758-763.

33. McLean RR, Jacques PF, Selhub J, et al. (2004) Homocysteine as a predictive factor for hip fracture in older persons. $N$ Engl J Med 350, 2042-2049.

34. Commission Directive 2008/100/EC of 28 October 2008 amending Council Directive 90/496/EEC on nutrition labeling for foodstuffs as regards recommended daily allowances, energy conversion factors and definitions. 